\title{
38
}

\section{QoS characterization of the ATM Block Transfer mode with Instantaneous Transmission}

\author{
Gérard Hébuterne
}

Institut National des Télécommunications

91011, Evry, France. Tel: (+33)160764583 gerard.hebuterne@intevry.fr

\begin{abstract}
Using the ATM technique to carry efficiently variable bitrate connections is a challenge which gives rise to numerous studies and proposals. Aside from open loop schemes such as Deterministic Bit Rate and Statistical Bit Rate, much attention is paid on procedures able at making profit of the characteristics of "elastic traffic". Here, the source is assumed to be able to adapt its behaviour to the network state.

The Available Bit Rate ABR is probably the most popular solution to have been proposed. In this paper, we focuse on another one, currently under study in the ITU-T, namely the ATM Block Transfer mode with Instantaneous Transmission.

As for any "best effort" scheme, the network may not indefinitely allow new connections without any control. We propose to make use of the time to send a burst as the QoS criterion. We give a simple model of the procedure which captures the main characteristics of the source and network interaction, and allows an estimation of the criterion. Especially, reattempts are included in the source description, and the QoS is then specified in terms of delay and available capacity.

This work was prepared during a visit to INRS-Telecom (Montreal, Canada)
\end{abstract}

\section{Keywords}

ATM, QoS, ABT, reattempts

\section{INTRODUCTION}

Using the ATM technique to carry efficiently variable bitrate connections is a challenge which gives rise to numerous studies and proposals. The case where the service has stringent requirements in terms of cell delay variation and instantaneous bitrate is dealt with by open loop schemes - the source declaring its characteristics and requirements once and for the whole call 
duration. ITU-T and the ATM-Forum have defined the Statistical Bit Rate SBR (respectively, Variable Bit Rate VBR) transfer mode to take account of these classes of services (ATM Forum 1996, ITU-T 1996).

Closed loop schemes are seen as more promising in terms of network efficiency. Here, the source is assumed to have far less strict Quality of Service (QoS) requirements. Especially, the source is able to react to information messages coming from the network, giving rise to cooperative schemes. Such sources able at varying their traffic without interrupting the connection are refereed to as elastic applications. The dialog between the source and the network is carried out through the so-called Resource Management (RM) cells.

The best known example of reactive scheme is the Available Bit Rate mode (ABR) currently defined by the ATM-Forum (ATM Forum 1996). However, the description of the ABR mode makes it obvious that any implementation of the procedure should exhibit a high level of complexity, to say nothing of the numerous pending issues such as source behaviour control and parameter tuning. Another cooperative scheme has been proposed - see (Boyer et al. 1992a, Boyer et al. 1992b, Guillemin 1997) and is currently under consideration in the ITU-T working groups under the name ATM Block Transfer Capability $(A B T)$. It is expected that ABT leads to simpler implementation while achieving a fairly efficient use of the network resources. This should be evident from the description below, where the ABT mode appears as a kind of "piecwise DBR" mode.

Whichever scheme is considered, assessing the Quality of Service (QoS) its provides is a mandatory part of its specification. Even for services described as best-effort services, one has to be able to give a rough characterization of the QoS level the network achieves. This may be used e.g. to assess a nominal QoS level and to help in network dimensioning, or better to serve as a basis for elementary Connection Acceptance Control (CAC) procedures.

In this study, we focuse on a possible definition for the QoS attached to the $\mathrm{ABT} / \mathrm{IT}$ mode. We propose a model able at predicting its performance level and at the design of the associated CAC procedure.

The paper is organized as follows. To begin with, Section 2 describes briefly the $\mathrm{ABT} / \mathrm{IT}$ and its operating mode. The need for specification of the service provided and the analysis of the source requirements leads to a proposed means of describing the achieved QoS.

Section 3 presents the assumptions the model is built on. They concern the source behaviour, which is kept as elementary as possible, while taking account of the main feature of the typical services involved, and especially reattempts. The other assumptions concern the network behaviour and are intended at building a tractable model. The model is presented in Section 4.

The results are discussed in Section 5 through a few numerical examples. Some comments are given concerning the accuracy of the assumptions, and the use which can be made of them in order to build tentative CAC procedures. 


\section{THE ABT/IT TRANSFER MODE}

The ABT capability is based upon the principles of the Fast Reservation Protocol, see (Boyer et al. 1992a, Boyer et al. 1992b). In the basic operating mode $A B T / D T$, the source opens a DBR circuit, and submits to the network requests to vary the peak cell rate allocated to it. The request, if accepted, provokes a change in the parameters (peak rate) and the source may accordingly modify its sending bitrate.

The ABT/IT capability is a variant which aims at further simplifying the procedure by eliminating the need to buffer the increase in traffic while waiting for the answer to the request. Since most often a positive answer to requests for increase is expected, the ABT management module anticipates and begins sending at the new bitrate - with the possibility of a negative answer, in which case part of the traffic will be lost.

In this study the ABT/IT variant will be further explored.

\subsection{A brief description of $\mathrm{ABT} / \mathrm{IT}$}

The connection is set up with some value for the allocated peak bitrate. The value of the peak rate is refereed to as Block Cell Rate (BCR) in the ITU-T literature. Sources willing to modify their emission rate send RM cells requesting a new value for their BCR. Immediately after they begin sending information using the new value, without waiting for any acknowledgement. The case where the bitrate is decreased raises no difficulty. In case where the bitrate is increased, the possibility exists that a node along the path has no available resource to accomodate it. A part of the flow is then discarded, and a negative acknowledgment is sent back to the node. If the upgrade request succeeds, the flow eventually reaches its destination, and a positive acknowledgment is sent to the source.

The connection makes use of some flow control mechanism. Especially, the source cannot send a new burst before the previous one is positively acknowledged. The process which manages the ABT/IT connection keeps sending unsuccessful bursts until they are correctly received. The precise way the lost bursts are corrected is beyond the scope of the present work.

The simplicity of the ABT mode can be described by stating that it can be truly described as "piecewise DBR" (Guillemin 1997) - between two requests, the source behaves really according to the DBR scheme.

\subsection{QoS issues for ABT transfer mode}

There are two levels at which QoS issues must to be examined. At the cell level, since a connection obeying the ABT mode works exactly as a DBR 
connection (at least, once the negotiation for the requested bandwidth has ended successfully), no special discussion is needed here and the QoS can be specified just as for the DBR mode. Moreover, guaranteeing a given level of QoS requires no specific action from the network side.

At the "burst" level, "best effort" schemes (such as ABR or ABT) rely on the possibility to lower the instantaneous bitrate offered to the sources. The maximum delay (as measured by some quantile of the end-to-end delay distribution) would yield a poor characterization: due to the intrinsic rules of the procedure, the maximum delay has to be large, so that it would be of no practical use. In fact, the main trouble sources may encounter in a best effort network is a progressive collapse of their transmission capability, due e.g. to a continuous increase in the number of open and active connections. In the ABT (for both the DT and IT variants) this happens through the rejection of the "increase bandwidth requests". Services using such a procedure as ABT/IT are likely to complete it with some error control and correction through retransmission. As a consequence, the average time until the request is positively acknowledged is a realistic parameter for the source to estimate the level of service. This delay gives the source an upper bound of the rate at which it can enter data into the network.

It must be emphasized at that point that not only the request rejection probability would not be an accurate QoS criterion, but also that a loss model is quite inaccurate. A model with retrials is much more effective in describing the complete behaviour of the network and the traffic sources.

\section{ASSUMPTIONS AND NOTATIONS}

\subsection{Traffic and network models}

In order to give an estimate of the network performance, we build a simple model of the traffic pattern and source behaviour.

The sources are assumed to share a set of links of the same capacity $C$. All sources are of the ON/OFF type and make use of the ABT/IT service. They remain silent (no allocated bandwidth) until they generate a burst at a peak rate equal to $c$ (giving rise to a bandwidth request). This gives the simplest possible model for the process of request generation. The protocol unit in the ingress node manages the request and the acknowledgements. In order to simplify the discussion, the acknowledgments are assumed to be loss-free (one can assume they use a dedicated DBR VC).

The following events must be carefully distinguished:

- A request is the operation by which a source sends a data block to the destination.

- A trial is the event when the source makes an attempt to send data to the 
destination. The trial may fail: in this case it is followed by another trial, until the source receives a positive acknowledgment.

- Each trial gives rise to bursts sent successively on the links of the route.

The number of nodes (or equivalently of links) between the source and the destination of the connection defines the length of the route. In order to keep a model as simple as possible, all connections are assumed to have the same length $D_{0}$. However, unsuccessful trials have an effective length $D, D<D_{0}$. Figure 1 summarizes the definitions. The path has a length $D_{0}=3.4$ trials are necessary to process the request, and they give rise to 8 bursts.

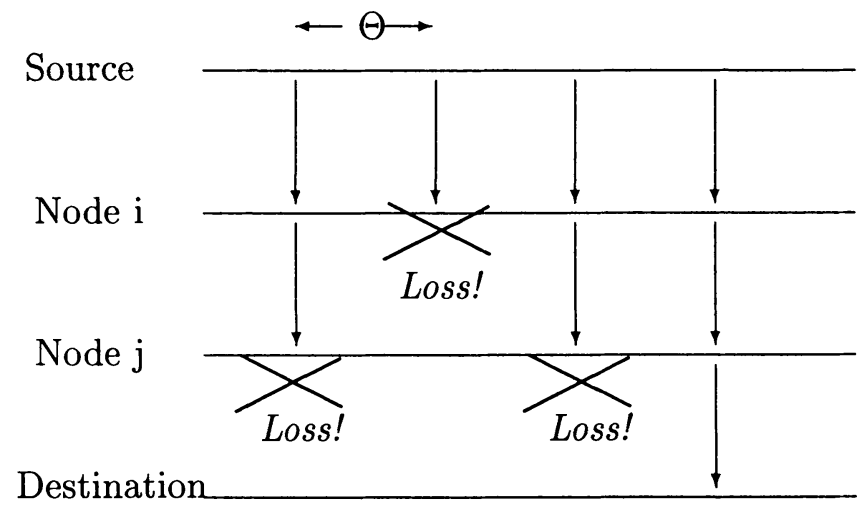

Figure 1 A request, made up with trials and bursts

We make the assumption that the traffic processes on the links are independent variables. The traffic is assumed to be uniformly distributed throughout the network so that all links are equally loaded. The model considers a particular link of the network. Let $\Lambda$ be the number of requests per time unit sent by all connections which go through the link under study. These requests originate from nodes at distance less than or equal to $D_{0}$.

\subsection{A summary of the notations}

- $C$ is the link capacity, $c$ is the peak rate at which the sources send data, so $R=C / c$ bursts can be accomodated in a link without any loss.

- $D_{0}$ : the length of the path from the source to the destination. Actually, since a trial may end up unsuccesfully, let $D$ denote the length of an unsuccessful trial. Let $D_{T}$ be the total number of bursts sent through all the links of the route in order to successfully send the request (in Figure 1, the first trial has $D=2$, etc., and $D_{T}=8$ ). 
- $T$ is the total time to send a burst. The geographic distances in the network are assumed to be negligible so that the data is available at the receiving end without additional transmission delay, and $T$ may be assumed as independent of $D_{0}$.

To further simplify the expressions and notations, it is assumed that there is no additional delay in intermediate nodes. An ATM cell entering the node is immediately available for being sent if needed. To summarize, if the source begins sending a burst at time $t$, the burst is available in the receiver at the distance $d$ at time $t+T$ (provided it has been successfully transmitted).

- When more than one trial is needed, the successive trials are time spaced by $\Theta$. This delay accounts for all processing delays through the route and for any additional delay requested by the correction procedure (see discussion on Section 6).

- $\Lambda$ is the arrival rate in number of requests per time unit from all the connections which seize the link under study.

- $p$ : the probability that a burst fails while accessing the link (due to shortage of capacity on the link under consideration which already accomodates $C / c$ bursts). With the assumptions on traffic homogeneity, $p$ is constant throughout the network. Moreover, we make the assumption that it does not depend on the rank of the trial.

- Let $\pi$ denote the probability that a trial fails. Successive links are assumed to behave independently, the relation between $\pi$ and $p$ is thus

$$
\pi=1-(1-p)^{D_{0}}
$$

Finally, Figure 2 displays the proposed model for performance evaluation of the $\mathrm{ABT} / \mathrm{IT}$ procedure. Unsuccessful trials give rise to reattempts which are dealt with by the Management Unit. The source is assumed to wait for the acknowledgement of a request before sending another one.

\section{PERFORMANCE EVALUATION}

\subsection{Behaviour of a request}

With $\pi$ as the failure probability of a trial, the probability that a request gives rise to $j$ trials (the last one successful) is $\pi^{j-1}(1-\pi)$. On the average $1 /(1-\pi)$ trials are needed.

Now, if a particular trial is to fail during its travel to the destination, the conditional probability that it reaches the $k$-th node is:

$$
P(D=k \mid \text { failure })=\frac{p(1-p)^{k}}{\pi} \quad k<D_{0},
$$




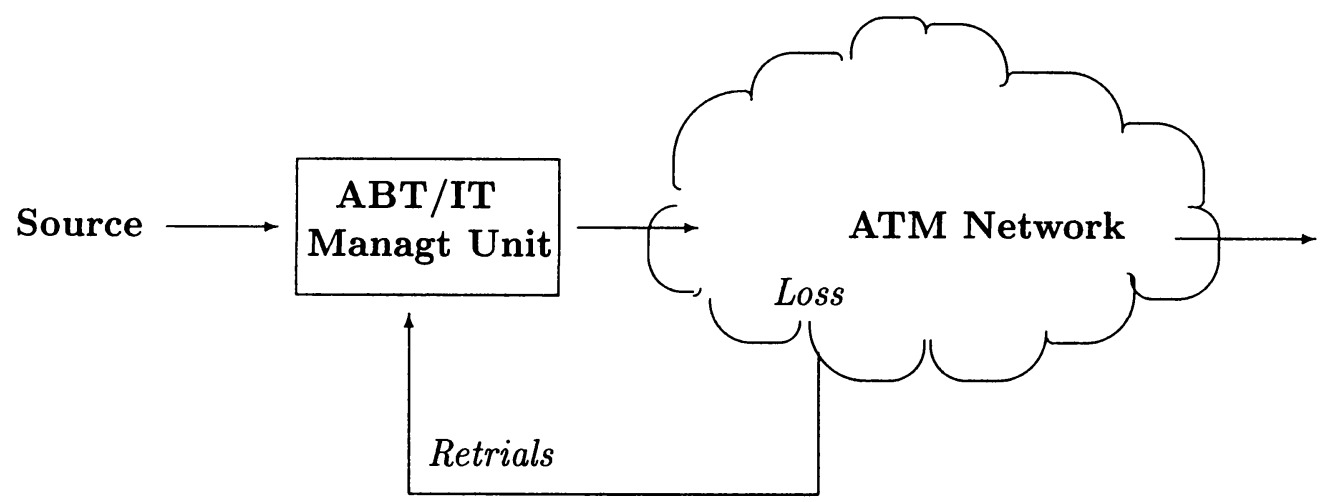

Figure 2 The traffic model with retrials

so that the mean length of failing paths is:

$$
\mathrm{E}(D \mid \text { failure })=\frac{1-p}{p}-\frac{D_{0}(1-p)^{D_{0}}}{\pi} .
$$

Finally, processing a request gives rise to a number of bursts sent in the network equal to $\mathrm{E}\left(D_{T}\right)$ :

$$
\mathrm{E}\left(D_{T}\right)=D_{0}+\frac{\pi}{1-\pi}\left(\frac{1-p}{p}-\frac{D_{0}(1-p)^{D_{0}}}{\pi}\right)=\frac{\pi(1-p)}{p(1-\pi)}
$$

It can be verified from eqn.(1) and (2) that $\lim _{p \rightarrow 0} \mathrm{E}\left(D_{T}\right)=D_{0}$.

\subsection{Model of the link}

The "link", or rather the multiplexing stage at the node egress, receives bandwidth requests at a rate $\Lambda$ per time unit from nodes at distance $D_{0}$ or less.

On the average, a request generates $\mathrm{E}\left(D_{T}\right)$ bursts inside the network. Since all links are assumed equally loaded, this amounts to share this traffic among the $D_{0}$ links of the path (as if each request was contributing to the load of each link of its path to the level $\left.\mathrm{E}\left(D_{T}\right) / D_{0}\right)$.

Each burst occupies a fraction of the link for a duration $T$. The traffic carried by the link under consideration is finally:

$$
A_{c}=\Lambda \times \frac{\mathrm{E}\left(D_{T}\right)}{D_{0}} \times T=\Lambda T \frac{\pi(1-p)}{p(1-\pi) D_{0}} .
$$


With $1-p$ as probability of success, the traffic offered to the link is

$$
A_{o}=\frac{\Lambda T \pi}{p(1-\pi) D_{0}} .
$$

Note that $\Lambda$ should increase with $D_{0}$. In an homogeneous network the ratio $\Lambda / D_{0}$ should have a value roughly constant with $D_{0}$.

In the case where no rejection never occurs (this would be the case if instead of being rejected the bursts were allowed to wait in the intermediate nodes), the traffics would always have their maximum value: $A_{o}=C / c$, and the network would thus achieve its greater efficiency. From the result already quoted: $\lim _{p \rightarrow 0} \mathrm{E}\left(D_{T}\right)=D_{0}$ one sees that the theoretical maximum capacity would then be: $(\Lambda T)_{M}=C / c$ as can be expected. The efficiency of the procedure may be expressed as $A_{o} /(\Lambda T)_{M}$.

Now, $1 /(1-\pi)$ trials are needed to successfully transmit the request. The successfull one takes a time $T$ while a delay $\Theta$ runs between unsussfull ones. This gives the transfer delay - or equivalently the capacity really offered to the sources:

$\mathcal{D}=T+\frac{\pi \Theta}{1-\pi}, \quad \mathcal{C}_{\text {eff }}=C \times(1-\pi)$.

\subsection{The rejection process}

In order to relate the loss ratio with the traffic process, we rely on the classical simplifying assumptions made for systems with reattempts (see e.g. (Hébuterne 1987). Each link is seen as being submitted a Poisson process resulting of the superposition of the fresh offer and of all successive bursts, as if the outcome of the reattempts were independent of the initial failing. Relation 4 gives the volume of the traffic offered to the link. The instantaneous level of the fresh offered traffic is independent of the number of requests in retrial process. So, the link is modeled according to the classical Erlang model. The loss ratio is given by the Erlang formula:

$p=\operatorname{Erl}\left(\frac{C}{c}, A_{o}\right)$.

Taken together, relations $(1,4,6)$ allow to solve for $p, \pi, A_{o}$ and the related variables.

\section{NUMERICAL ANALYSIS}

The efficiency of the ABT/IT procedure is measured by the available traffic the network may carry. Note that since the model assumes the rejected request 
can make reattempts for ever, there is no distinction between offered and carried end-to-end request traffic. The traffic carried by the links is the scarce resource which serves as a control variable.

In order to obtain significant results, the carried traffics are normalized using the maximum achievable value. That is, the parameter of interest is $A_{o} /(\Lambda T)_{M}$. Similarly, the traffic carried by the link is represented as the "link occupancy", that is the carried load, as given by equation (3), normalized to its maximum value $C / c$. This allows fair comparison between cases where e.g. the distance parameters are different.

The first set of curves (Figure 3) displays the influence of the relative size of the channel capacity and of the requests on the overall performance. The efficiency is the ratio $A_{o} /(\Lambda T)_{M}$ and the "link occupancy" is $c A_{c} / C$. Note that the ratio of the efficiency to the link occupancy is $p D_{0} / \pi$.

The higher the ratio $C / c$ the better in terms of maximum available capacity: ratios higher than 20 are probably to be expected, since anyway no CAC would allow realistic network occupancy for lower values. Efficiencies around 60 to $70 \%$ are achievable, with a collapse above a maximum value due to the contention. Note however that as opposed to the classical "collision" of the CSMA scheme, the contention leads to the destruction of only one of the 2 bursts.

The influence of the number of nodes is displayed in Figure 4. The performance degradate as the route lengthens, which favors networks with small diameters and accordingly large nodes. Note that however the influence of the length is not so high as the one of the ratio $C / c$.

At last, Figure 5 displays the variation of the additional end-to-end burst transfer delay. The delay is measured in units of $\Theta$, for route length $D_{0}=5$. For each curve, the horizontal line indicates the point where the efficiency has the maximum value. It is clear that, as long as the network is operated for services such that $C / c$ is not too small (say, greater than 20), the QoS, as measured by the delay, is quite satisfactory (delays due to retrials lower than $0.35 \Theta$, here).

This analysis shows that the procedure may exhibit good performance, as long as it is operated on networks with small diameters (connections of moderate length) and for services requiring moderate bitrates (so that $C / c$ remains high). These conditions, however, are typically the ones under which any statistical multiplexing procedure is expected to work. Provided these conditions hold, the network may achieve high efficiency (effective capacity offered to the sources higher than $60 \%$ ).

The procedure has to be complemented by some control process aiming at avoiding the operating point to move to the collapsing zone of curves (Figures 3,4$)$. The ABT/IT Management Unit should make use of the time out procedures which are known to provide stable operation of the CSMA/CD scheme. 


\section{COMMENTS AND CONCLUSIONS}

The model used in the previous Sections makes a few assumptions which call for comments. Probably the strongest simplifying assumption is to consider that all bursts have the same failure probability $p$. Obviously, one would expect a higher loss probability for the second trial, for instance, than for the first one. To state it another way, the loss probability of a burst depends on the time elapsed since the last loss. Considering that $p$ does not depend on the rank of the trial amounts to say that the interval between successive trials $\Theta$ is large as compared with the typical time scale of the multiplexing stage say, as compared with the burst duration.

The derivation assumes independence between the number of pending requests in the network and the rate at which new ones arrive. In fact, since the link under study carries the traffic on a fixed number of connections, a more realistic model would be to consider the requests as being generated by a finite number of sources (Engset like traffic). The Poisson assumption corresponds to the asymptotic worst case, so that the results are conservative.

Finally, the traffic configuration deserves a few comments. The source traffic pattern consisting of bursts (On/Off source) is simplistic, and actual ATM sources can be fairly more various. Especially, the sources the ABR mode considers require a minimum bitrate (MCR), which would be quite easy to take account of in the model without degradating its accuracy. Stepwise traffic can be considered too (the source keeps on sending traffic at a given BCR and updates it now and then). While this configuration would modify the source behaviour, the model proposed still applies provided the product $\Lambda T$ is given the right meaning.

The results show the $\mathrm{ABT} / \mathrm{IT}$ procedure as a candidate to the provision of a "best effort" based multiplexing scheme. The dependence in the distance (length of the route measured in number of intermediate nodes) may be alleviated by organizing the network in segments ended by virtual sources destinations, just as it has been proposed for the ABR mode. Concerning the dependence on the ratio $C / c$, which conditions mainly the global efficiency, the results favor large ratios, and show that values below 20 are to be avoided.

This paper has proposed a way to describe the QoS offered by a best effort service. Working on the Web gives an idea of the service provided by completely un-controlled network. This makes it obvious the need to implement some connection admission function based upon the mean transfer delay. From the results presented here such a CAC procedure would proceed by limiting the number of simultaneous connections. The full design of the procedure is however still to be undertaken. The models presented give the means to estimate its performance level. 


\section{REFERENCES}

The ATM Forum: UNI Specification, Version 4.0, June 1996.

P. Boyer, F. Guillemin, M. Servel, J.P. Coudreuse (1992) Spacing Cells Protects and Enhances Utilization of ATM links. IEEE Communications Magazine, September 1992.

P. Boyer, D. Tranchier (1992) A reservation Principle with Application to the ATM Traffic Control. Computer Networks and ISDN Systems, 24, 321-334, 1992 .

F. Guillemin: ATM Block Transfer Capability vs. Available Bit Rate Service. European Transactions on Telecommunications, 8, No1, January 1997.

G. Hébuterne: Traffic flows in switching systems. Artech House, 1987.

ITU-T Recommendation I.371: Traffic Control and Congestion Management.

Geneva, June 1996.

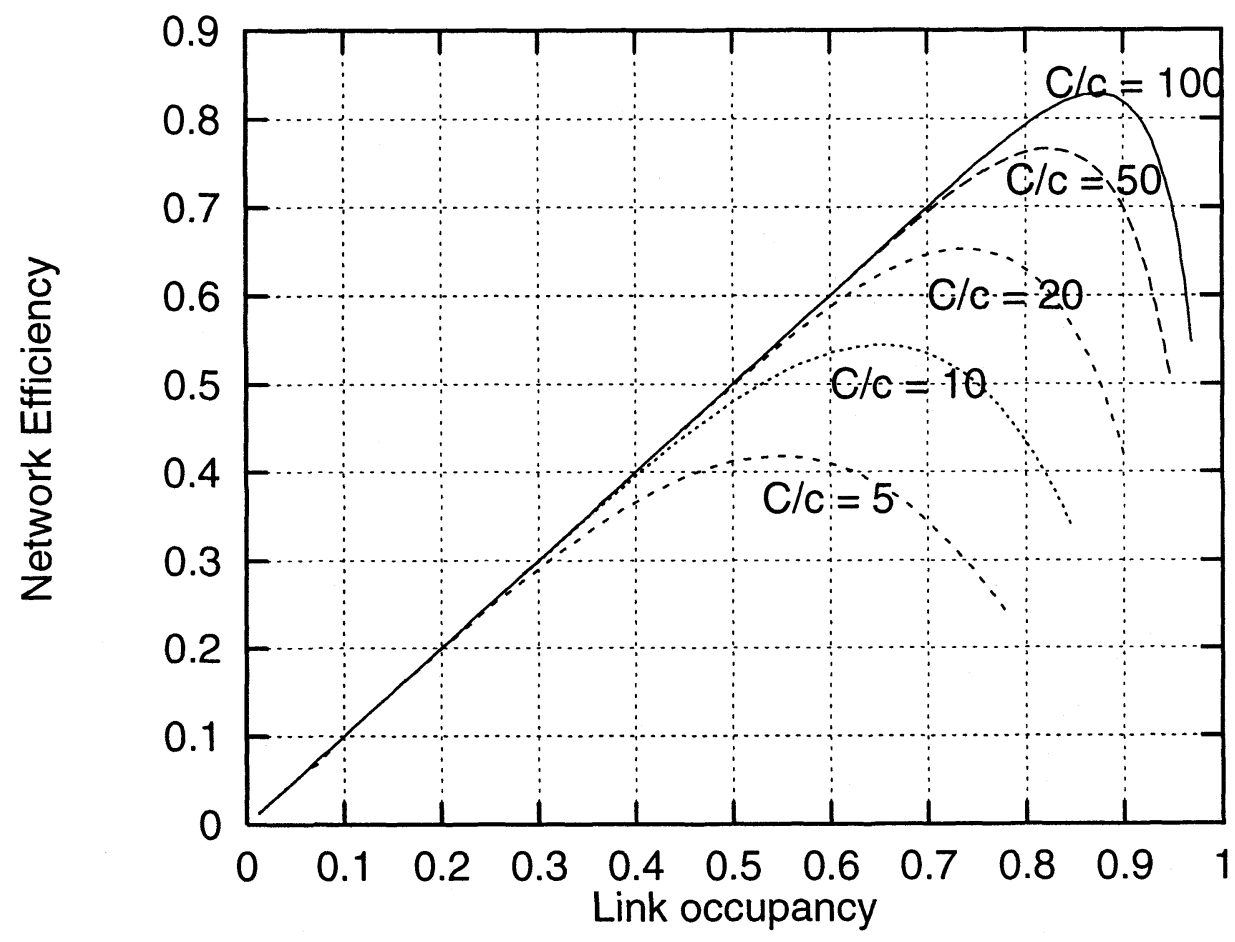

Figure 3 Relation between offered load and effective link load. Influence of the request size. The curves are drawn for a connection length $D_{0}=5$ 


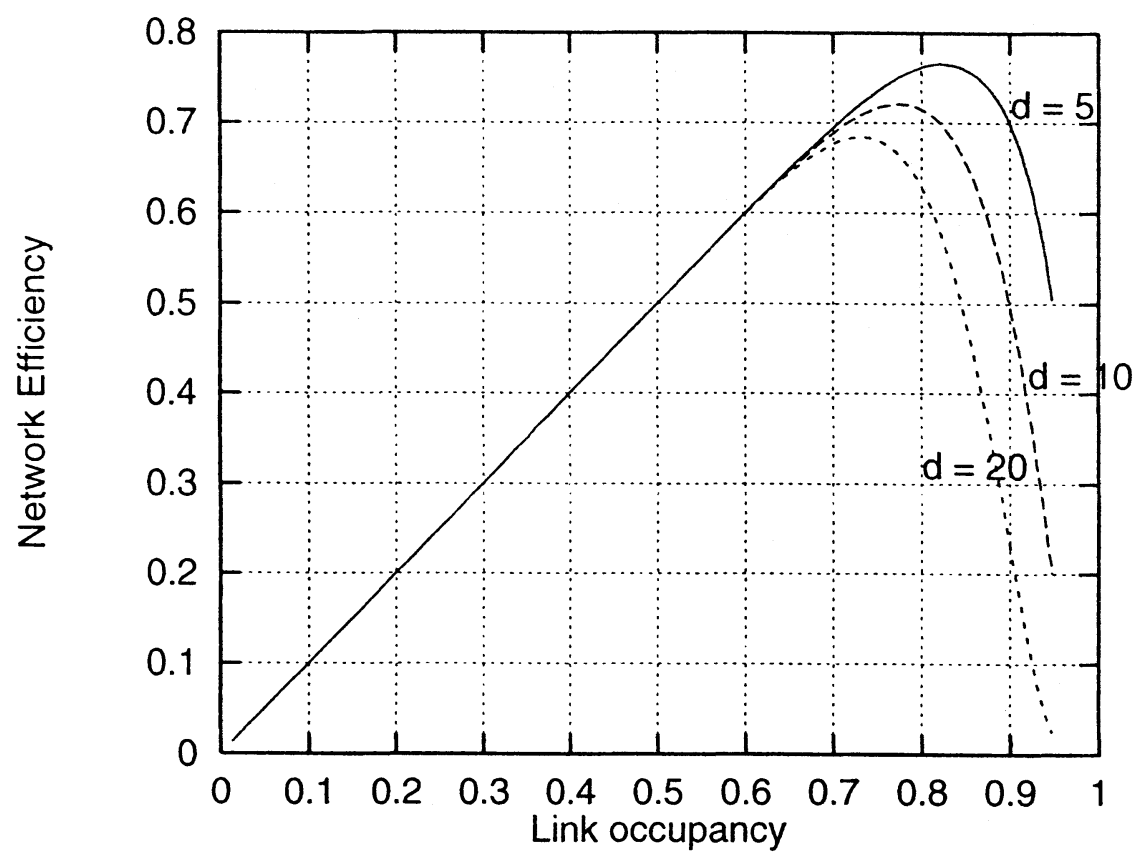

Figure 4 Offered load vs Effective link load. Influence of route length $(C / c=$ $50)$.

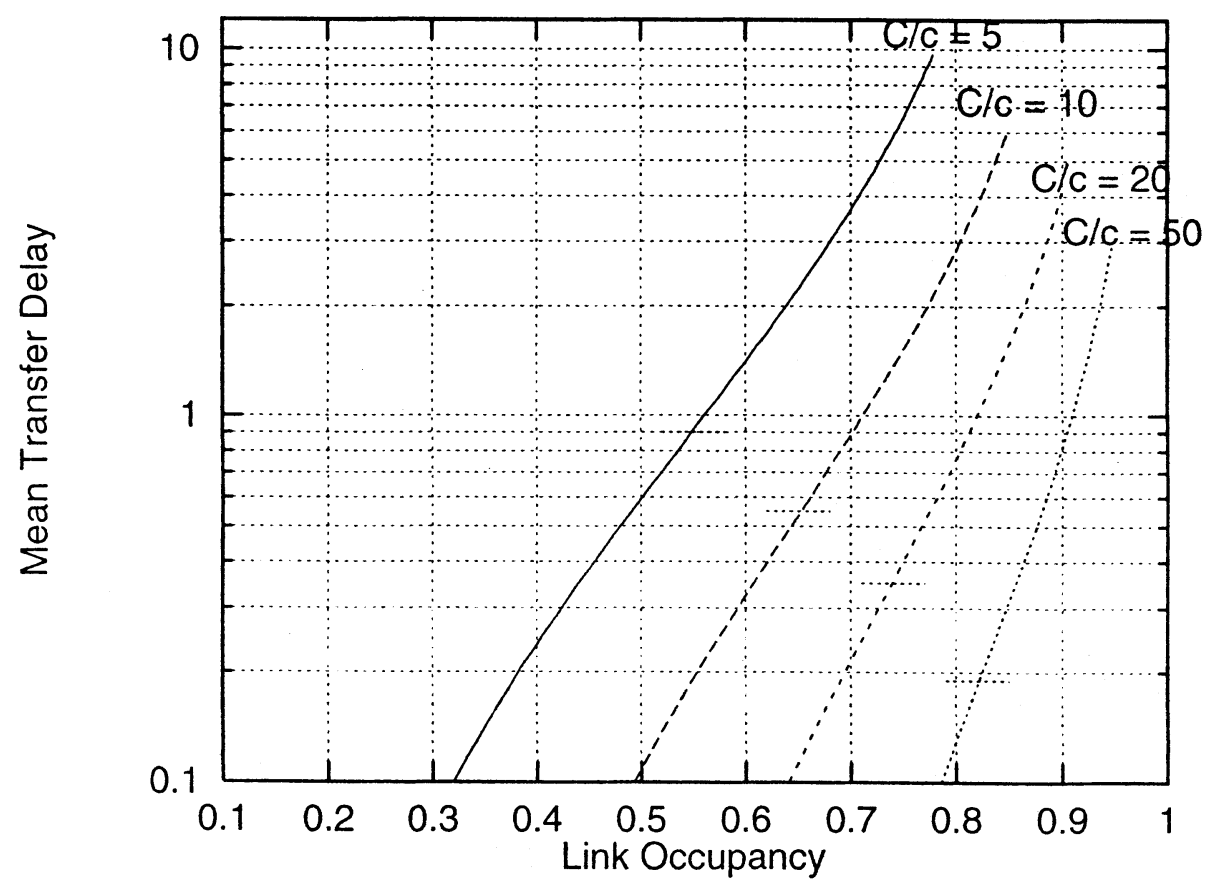

Figure 5 Mean Transfer Delay vs Effective link load $\left(D_{0}=5\right)$. 